\title{
Real world effectiveness of primary implantable cardioverter defibrillators implanted during hospital admissions for exacerbation of heart failure or other acute co-morbidities: cohort study of older patients with heart failure
}

\author{
Chih-Ying Chen, ${ }^{1}$ Lynne Warner Stevenson, ${ }^{2}$ Garrick C Stewart, ${ }^{2}$ Deepak L Bhatt, ${ }^{2}$ Manisha Desai, ${ }^{3}$ \\ John D Seeger,' Lauren Williams, ${ }^{1}$ Jessica J Jalbert, ${ }^{1}$ Soko Setoguchi ${ }^{4}$
}

\author{
'Division of \\ Pharmacoepidemiology and \\ Pharmacoeconomics, \\ Department of Medicine, \\ Brigham and Women's Hospital \\ and Harvard Medical School, \\ Boston, MA 02120, USA \\ ${ }^{2}$ Division of Cardiovascular \\ Medicine, Brigham and \\ Women's Hospital and Harvard \\ Medical School, Boston, MA \\ 02115, USA \\ ${ }^{3}$ Quantitative Sciences Unit, \\ Stanford University School of \\ Medicine, Palo Alto, CA 94305 , \\ USA \\ ${ }^{4}$ Duke Clinical Research Institute, \\ Durham, NC 27705, USA \\ Correspondence to: \\ S Setoguchi \\ soko.setoguchi@duke.edu \\ Additional material is published \\ online only. To view please visit \\ the journal online (http://dx.doi. \\ org/10.1136/bmj.h3529) \\ Cite this as: BMJ 2015;351:h3529 \\ doi: 10.1136/bmj.h3529 \\ Accepted: 16 Jun 2015
}

\section{ABSTRACT}

\section{OBJECTIVES}

To examine the effectiveness of primary implantable cardioverter defibrillators (ICDs) in elderly patients receiving the device during a hospital admission for exacerbation of heart failure or other acute co-morbidities, with an emphasis on adjustment for early mortality and other factors reflecting healthy candidate bias rather than the effect of the ICD.

DESIGN

Retrospective cohort study.

SETTING

Linked data from the Centers for Medicare and Medicaid Services and American College of Cardiology-National Cardiovascular Data Registry ICD registry, nationwide heart failure registry, and Medicare claims data 2004-09. POPULATION

23111 patients aged $\geq 66$ who were admitted to hospital for exacerbation of heart failure or other acute co-morbidities and eligible for primary ICDs.

\section{MAIN OUTCOME MEASURES}

All cause mortality and sudden cardiac death. Latency analyses with Cox regression were used to derive crude

\section{WHAT IS ALREADY KNOWN ON THIS TOPIC}

The benefit of primary implantable cardioverter defibrillators (ICDs) has been shown in outpatients with symptoms of stable mild-to-moderate heart failure It is unclear how the impact of primary ICDs in preventing sudden cardiac death translates to overall survival benefits among elderly patients who receive the devices during acute admissions for exacerbation of heart failure or other acute co-morbidities

Evaluating survival benefits of ICDs in the real world setting without accounting for healthy candidate effect could overestimate its effectiveness

\section{WHAT THIS STUDY ADDS}

This study used multiple analytical approaches (latency analysis and adjustment for high dimensional propensity scores) to account for healthy candidate bias in assessing effectiveness of ICDs with observational data

After adjustment for healthy candidate bias and confounding, the benefits of primary ICD therapy shown in previous trials were not present in elderly patients who received the device during admission for exacerbation of heart failure or other acute co-morbidities

The trend was similar in subgroups of patients aged under 80 , in both sexes, and in racial subgroups

There was a trend towards benefit in reducing mortality or sudden cardiac death in patients who had had a myocardial infarction more than 40 days previously, left bundle branch block, or low serum $B$ type natriuretic peptide

hazard ratios and hazard ratios adjusted for high dimension propensity score for outcomes after 180 days from index implantation or discharge.

RESULTS

Patients who received an ICD during a hospital admission had lower crude mortality risk than patients who did not receive an ICD (40\% v60\% at three years); however, with conditioning on 180 day survival and with adjustment for high dimension propensity score, the apparent benefit with ICD was no longer evident for sudden cardiac death (adjusted hazard ratio 0.95, $95 \%$ confidence interval 0.78 to 1.17 ) and had a diminished impact on total mortality $(0.91,0.82$ to 1.00). There were trends towards a benefit with ICD in reducing mortality or sudden cardiac death in patients who had had a myocardial infarction more than 40 days previously, left bundle branch block, or low serum B type natriuretic peptide; however, these trends did not reach significance.

CONCLUSION

After adjustment for healthy candidate bias and confounding, the benefits of primary ICD therapy seen in pivotal trials were not apparent in patients aged 66 or over who received ICDs during a hospital admission for exacerbation of heart failure or other acute co-morbidities. Future research is warranted to further identify subgroups of elderly patients who are more likely to benefit from ICDs. Recognition of those patients whose dominant risk factors are from decompensated heart failure and non-cardiac co-morbidities will allow better focus on ICDs in those patients for whom the device offers the most benefit and provides meaningful prolonging of life.

\section{Introduction}

The most recent worldwide survey of cardiac pacing and implantable cardioverter defibrillators (ICDs) from 2009 reported a large global rise in the use of these devices. ${ }^{1}$ The United States is the world's largest consumer of ICDs, with 133262 implants (or 434 new implants per one million people), which was 1.5 times the rate of the world's second largest implanter. ${ }^{1}$ Review of US nationwide data on ICD implantation has shown that real world recipients are typically older than patients in previous trials, with a median age of $74,{ }^{2}$ similar to reports from other countries. ${ }^{3}$ As the population ages, the number of elderly patients considered for ICD implantation worldwide will most likely increase. ${ }^{45}$ 
The benefit of primary ICD therapy in landmark trials was shown in patients with heart failure in their $60 \mathrm{~s}^{67}$ The results from these trials, however, might not directly apply to older populations. Real world recipients of ICDs generally have more non-cardiac co-morbidities. $^{28}$ Furthermore, primary ICD trials were conducted in outpatients with symptoms of stable mild-to-moderate heart failure ${ }^{67}$ About a third of older recipients of ICDs have undergone an implantation during a hospital admission for exacerbation of heart failure or other acute co-morbidities. ${ }^{9}$ In patients with chronic heart failure, the early post-discharge period after an acute admission is associated with a high risk of mortality, during which progressive heart failure is the most likely cause of death. ${ }^{10}$ It is therefore unclear how the impact of primary ICDs on the prevention of sudden cardiac death translates to overall survival benefits among elderly patients who received the devices during acute admissions.

We examined the effectiveness of ICDs outside the previously studied populations in ICD trials. Our target population was elderly patients who received the device during acute admissions for exacerbation of heart failure or other acute co-morbidities. Assessment of the potential impact on survival with real world data requires caution because of healthy candidate bias. This type of selection bias could arise when patients at high risk of complications or deemed to be too sick to benefit are not selected for an ICD, and when patients are less interested in preventing sudden death because of the existing burden of other chronic illness. Evaluation of the clinical effectiveness of ICDs without consideration of the healthy candidate effect could overestimate its benefit. ${ }^{11}$ We therefore used specific design and analytic approaches to account for this bias when assessing the clinical effectiveness of ICDs implanted during admission for exacerbation of heart failure or other acute causes among elderly patients and potential differences in the effectiveness of ICDs by demographic and clinical characteristics. This included adjustment for mortality during the first 180 days, a period during which previous trials have shown no benefit of ICDs. ${ }^{67}$

\section{Methods}

\section{Data sources}

We conducted a retrospective cohort study using the ICD registry of the Centers for Medicare and Medicaid Services (CMS) (2005-08); ICD registry of the American College of Cardiology-National Cardiovascular Data Registry (ACC-NCDR) (2005-08); a nationwide heart failure registry aggregated from several quality improvement and accreditation programs, including the American Heart Association's Get With the Guidelines program (2005-08); and Medicare institutional and non-institutional claims (2004-09).

\section{ACC-NCDR and CMS-ICD registry}

The Medicare/Medicaid ICD registry is a subset of the American College of Cardiology-National Cardiovascular Data Registry's ICD registry, which is the sole repository for data on ICD implantation for Medicare beneficiaries. ${ }^{212-14}$ Hospital personnel enter data on the registry under routine quality control review. ${ }^{15}$ The registry includes information on patient history, clinical characteristics, drugs, facility information, provider information, indications for ICD, device information, and inpatient complications.

\section{Heart failure registry}

The data from the national clinical registries for patients with heart failure were aggregated from several quality improvement and accreditation programs managed by Outcome Sciences using common data elements, data clarification procedures, and quality assurance practices. The aggregate database includes data from over 800 US hospitals in 50 states with close to 300000 patients with a primary diagnosis of heart failure. The dataset has been successfully used to assess quality of care outcomes in patients with heart failure. ${ }^{16}{ }^{17}$ Information in the registry includes demographics, characteristics of heart failure, cardiac and non-cardiac medical history, laboratory data, vital signs, findings on relevant physical examinations, drugs on admission and at discharge, and other relevant treatment/procedures before and during admission.

\section{Medicare institutional and non-institutional files}

Medicare is the national health insurance program administrated by the US government. Most of its beneficiaries are aged 65 or older. The Medicare institutional and non-institutional files contain data on final claims submitted by healthcare providers. Main information contained in those files includes diagnosis and procedures, dates of service, reimbursement amount, provider identifiers, and demographic information on beneficiaries. Appendix 1 provides more details of these data sources.

\section{Data linkage}

We linked the combined ICD registry and the heart failure registry to Medicare claims data using four non-unique identifiers: date of birth, sex, admission date for implantation of the ICD, and provider identifiers, which is described in detail elsewhere ${ }^{18}$ and in appendix 1. Briefly, we validated this linkage among the subset of 196923 patients who had a unique identifier in the ICD registry. Our linkage using non-unique identifiers yielded 98\% specificity, 95\% sensitivity, and a $98 \%$ positive predictive value compared with the linkage method using both non-unique and unique identifiers. $^{18}$

\section{Study population}

Our study population consisted of elderly patients with heart failure with and without ICDs who had acute hospital admissions for heart failure or any co-morbidities and were considered eligible for ICD therapy for primary prevention. The primary cohort consisted of older patients who were covered by Medicare and who could be linked to either the ICD or heart 
failure registry. The secondary cohort consisted of older patients who were nested within the heart failure registry linked to Medicare data. To ensure their eligibility for primary ICD therapy, all study patients were required to have an ejection fraction of $\leq 35 \%$ at the time of admission. We excluded patients with cardiac arrest or sustained ventricular tachycardia, for whom ICDs would be secondary prevention. To ensure our study patients had a sufficient look-back period for assessing pertinent covariates, we required that patients had health insurance coverage for one year before the index procedure or admission. We also required patients to be aged $\geq 66$ to ensure everyone had a one year look-back period. The exposure status (ICD implantation) was defined as a patient having a record of ICD implantation in the ICD registry. Lastly, we excluded patients who received cardiac resynchronization therapy with a defibrillator (CRT-D) as these patients met additional criteria for this indication. ${ }^{19-21}$ The information on the type of implanted device was obtained from the ICD registry.

\section{Outcomes}

Our primary outcome was all cause mortality. The date of death was obtained from the Medicare beneficiary summary file. The secondary outcome was sudden cardiac death, defined by using a previously validated algorithm (positive predictive value $87 \%$ ). ${ }^{22}$ The designation of sudden cardiac death was made if the patient was not staying at a terminal institution (that is, hospital or nursing home) on the date of death, their code for underlying cause of death was consistent with sudden cardiac death (see appendix 2), and they did not have a "terminal procedure inconsistent with unresuscitated cardiac arrest, such as radiology, thrombolysis or general anesthetic." 22 The cause of death was obtained from the National Death Index ${ }^{23}$ (appendix 3). We obtained index data on all the patients with an ICD and a randomly selected sample (70\%) of those without in the primary cohort.

\section{Latency analyses}

We used latency analysis to adjust for potential healthy candidate bias, analogous to the healthy worker effect. ${ }^{24-26}$ Follow-up began after a prespecified latent period after the index date. In the current study, the index date was the date of implantation for the ICD group or the date of discharge for those without an ICD. As previous trials have shown that survival benefits of ICDs are not apparent until 1-1.5 years after implantation, ${ }^{67}$ we used latency periods of 180 days (primary latency period) and 365 days (secondary latency period). All patients were followed until the occurrence of an outcome event (death or sudden cardiac death) or the end of the study period (31 December 2009).

\section{Statistical analyses}

Patient characteristics were described as percentages for categorical variables by ICD exposure status. Medians and interquartile ranges or means and standard deviations were used for continuous variables.
We plotted observed mortality by ICD exposure status using Kaplan-Meier estimates. Cox regression was used to derive crude hazard ratios and hazard ratios adjusted for high dimensional propensity score for outcomes using the groups without an ICD (that is, older people admitted with equivalent indication for a primary ICD but who did not receive an ICD) as a reference.

We used the high dimensional propensity score methods to adjust for surrogates of unmeasured factors to overcome residual confounding. The algorithm was used to thoroughly screen Medicare claims data to identify covariates that could collectively be surrogates for unobserved factors influencing the patient selection for ICDs. ${ }^{27}$ This allows for maximum control of potential confounders given the available information in our data sources. For example, although we did not have information on NYHA class for heart failure, we can adjust for this based on other available proxies for severity of heart failure in the dataset, including numbers of previous hospital admissions for heart failure, ejection fraction, blood pressure, B type natriuretic peptide, or co-morbidities. To calculate high dimensional propensity scores, we used data from the year before the index date. The 200 most common codes in each data dimension were identified, from which 500 likely confounders were selected based on their prevalence and potential for confounding in the study population. The scores at the index date were derived from predicted probabilities from logistic regression models containing all of the empirically identified covariates and several predefined variables: demographic characteristics; cause of index admission, admission source, admission type, and diagnostic/laboratory test results for ejection fraction, systolic blood pressure, serum sodium, serum B type natriuretic peptide, and estimated glomerular filtration rate. ${ }^{28}$

We handled variables with missing values (such as systolic blood pressure, serum sodium, B type natriuretic peptide, and creatinine) by multiple imputation $^{29}$ and assumed an underlying multivariate normal distribution. Our analysis was based on five imputed datasets in which the imputation model included all variables in the outcome model (ICD use outcomes and potential confounders) as well as variables related to missingness (see appendix 4 for potential predictors of missing values included in the imputation model). We repeated all analyses in the secondary cohort.

\section{Subgroup analyses}

We assessed the heterogeneity of the effects of ICD by demographic characteristics (age, sex, and race) and three clinical characteristics (history of myocardial infarction, ${ }^{3031}$ presence of left bundle branch block, ${ }^{32} 33$ and type natriuretic peptide concentration at index admission) using separate Cox models in each subgroup.

Age was categorized in four groups in five year increments. We used a previously validated claim 
based definition to identify myocardial infarction ${ }^{34}$ and classified this population into three groups: recent myocardial infarction (one or more myocardial infarctions within the 40 days before the index date), old myocardial infarction (one or more myocardial infarctions 41-365 days before the index date), and no myocardial infarction within 365 days before the index admission. We also used a claim based definition to identify patients with left bundle branch block within 365 days before the index date (ICD-9 (international classification of diseases, ninth revision, clinical modification), $426.2 x$ or $426.3 x$ ). We required the diagnosis of left bundle branch block to be made during a hospital admission. We classified our patients into two groups according to B type natriuretic peptide concentration (low v high) using a cutoff value of $800 \mathrm{ng} / \mathrm{L}$. $^{35-37}$

We adjusted for potential confounding using the high dimensional propensity score estimated from the entire cohort. ${ }^{38}$

\section{Sensitivity analyses}

We repeated all analyses in a subset of the population with complete laboratory data and a subset of the population matched on high dimensional propensity score (that is, the high dimensional propensity score matched analyses). This was done to assess the impact of the

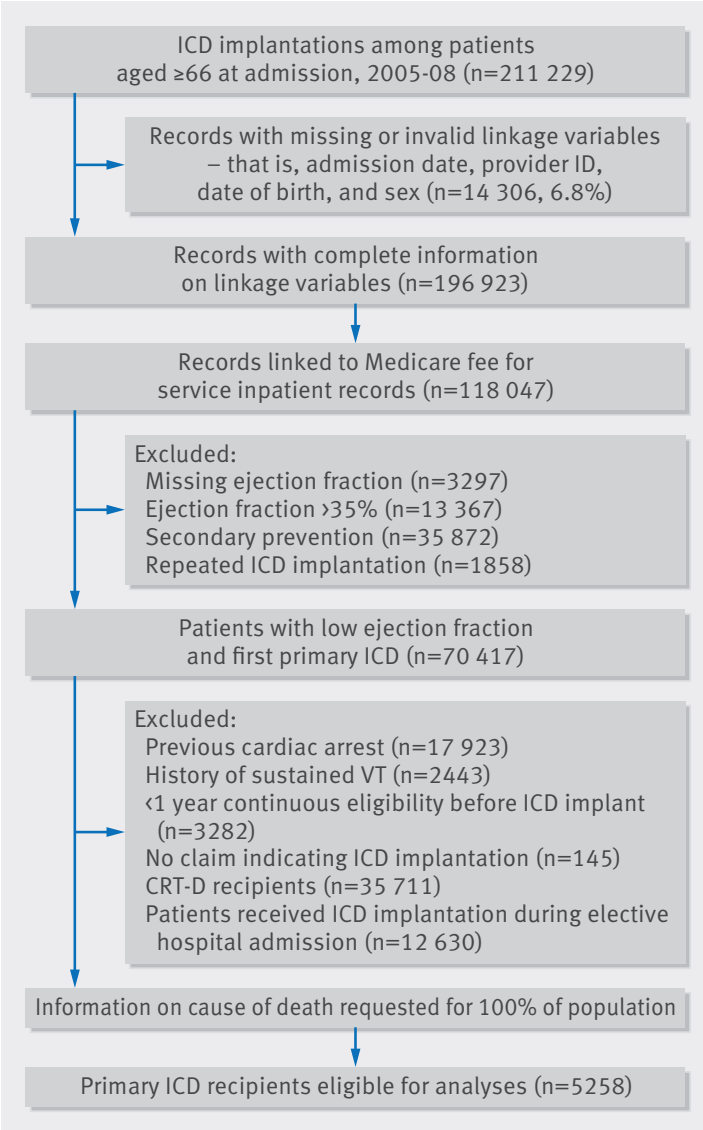

Fig 1 | Identification of study population of patients with heart failure with implantable cardioverter defibrillator (ICD) missing data assumption on our findings and the robustness of the adjustment using the high dimensional propensity score.

All analyses were conducted with SAS 9.2 (SAS Institute, Cary, NC).

\section{Results}

Study population and characteristics

We identified a cohort of 23111 patients with heart failure (5258 with an ICD and 17853 without) who met eligibility criteria (figs 1 and 2). For over $90 \%$ of the patients, the diagnosis that led to the index admission was heart failure or other cardiac causes. Patients with ICDs were younger and were more likely to be men than patients without ICDs. They also had a lower ejection fraction, more previous admissions for cardiac diseases, and more physician visits, and their heart failure was more likely to have an ischemic cause. Patients with ICDs also had a higher prevalence of non-cardiac admissions, chronic kidney disease, metastatic cancer, lower estimated glomerular filtration rate, and higher $B$ type natriuretic peptide (table1). These findings were similar in the secondary cohort (appendix 5).

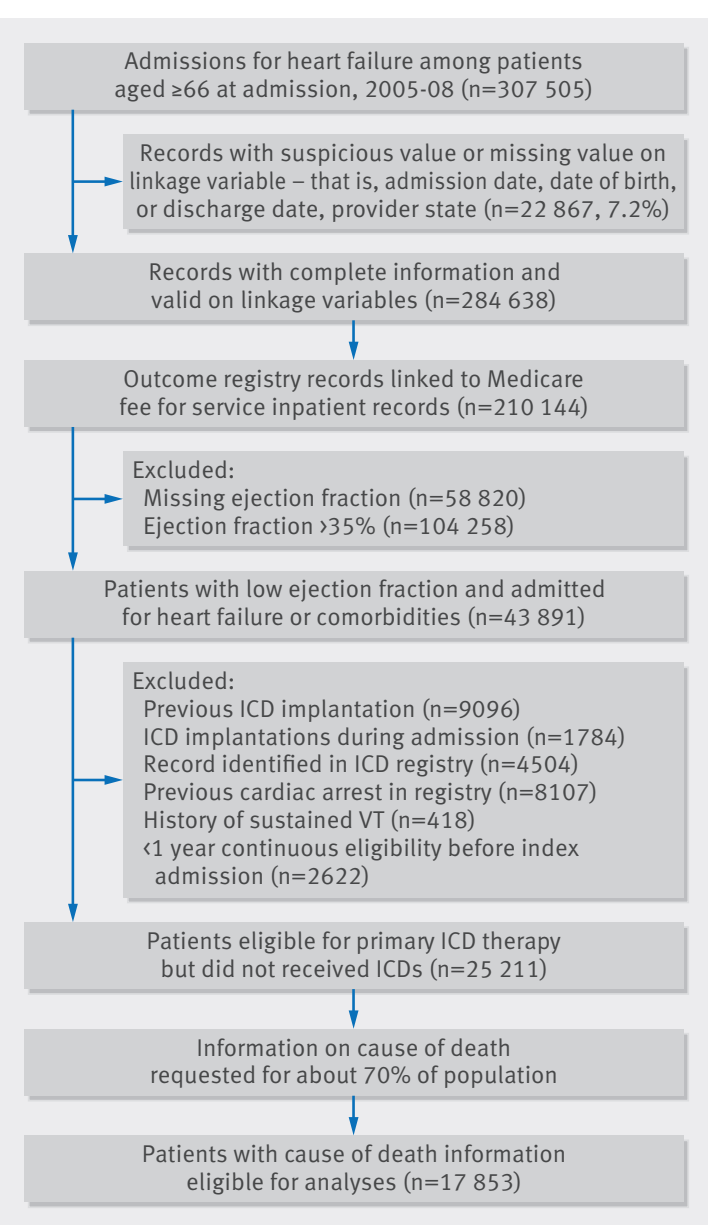

Fig 2 Identification of study population of patients with heart failure without implantable cardioverter defibrillator (ICD) 
Crude mortality risks and Kaplan-Meier curves

During follow-up (average 2.8 years, range 1 day-5 years), 12293 (53\%) patients died. The crude mortality risk among our Medicare population admitted to the hospital was 34\% (95\% confidence interval 33\% to 35\%) at one year and $56 \%$ (55\% to $57 \%$ ) at three years. The mortality curves for the patients with and without ICDs (fig 3) began to diverge immediately after ICD implantation $(2.4 \%(2.0 \%$ to $2.8 \%) v 12.7 \%(12 \%$ to $13 \%)$ at 30 days). Crude mortality at one year was lower for ICD recipients than for eligible patients without an ICD (18\% (17\% to $19 \%)$ v 39\% (38\% to $40 \%$ ) at one year and $40 \%(38 \%$ to $41 \%) v 60 \%(60 \%$ to $61 \%)$ at three years). However, the crude mortality in these hospitalized Medicare patients with an ICD at one year was similar to

\begin{tabular}{|c|c|c|c|}
\hline & No ICD $(n=17853)$ & ICD $(n=5258)$ & $P$ value \\
\hline Mean (SD) age (years) & $80.0(7.8)$ & $75.5(6.0)$ & $<0.001$ \\
\hline Men & $9321(52)$ & $3763(72)$ & $<0.001$ \\
\hline White & $15068(84)$ & $4392(84)$ & 0.13 \\
\hline Median (IQR) ejection fraction (\%) & $29(20-33)$ & $25(20-30)$ & $<0.001$ \\
\hline Median (IQR) Charlson scores & $3(1-4)$ & $3(1-4)$ & 0.68 \\
\hline \multicolumn{4}{|l|}{ Patients with $\geq 1$ hospital admission by cause: } \\
\hline Any causes & $9168(51)$ & $2912(55)$ & $<0.001$ \\
\hline Heart failure & $2844(16)$ & $1139(22)$ & $<0.001$ \\
\hline Myocardial infarction (MI) & $203(1)$ & $84(2)$ & 0.01 \\
\hline Non-Ml ischemic heart disease & 104 (1) & $74(1)$ & $<0.001$ \\
\hline Other cardiac disease & $51(0)$ & $39(1)$ & $<0.001$ \\
\hline Non-cardiac causes & $6501(36)$ & $1673(32)$ & $<0.001$ \\
\hline Mean (SD) No of prior outpatient visits & $10.3(9.4)$ & $11.7(9.3)$ & $<0.001$ \\
\hline$\geq 1$ prior outpatient visit & $16167(91)$ & $4974(95)$ & $<0.001$ \\
\hline$\geq 1$ prior skilled nursing facility admission & $2679(15)$ & $375(7)$ & $<0.001$ \\
\hline Heart failure due to ischemic causes & $14165(79)$ & $4587(87)$ & $<0.001$ \\
\hline Any cerebrovascular disease & $3860(22)$ & $1224(23)$ & 0.01 \\
\hline Hemorrhagic stroke & $198(1)$ & $93(2)$ & $<0.001$ \\
\hline Ischemic stroke & $1376(8)$ & $462(9)$ & 0.01 \\
\hline Transient ischemic attack & $1175(7)$ & $424(8)$ & $<0.001$ \\
\hline Other cerebrovascular disease & $2741(15)$ & $816(16)$ & 0.78 \\
\hline Peripheral vascular disease & $4147(23)$ & $1279(24)$ & 0.10 \\
\hline Dementia & $3503(20)$ & $584(11)$ & $<0.001$ \\
\hline Depression & $2821(16)$ & $662(13)$ & $<0.001$ \\
\hline Any liver disease & $1118(6)$ & $323(6)$ & 0.77 \\
\hline Gastrointestinal ulcer/bleeding & $2755(15)$ & $736(14)$ & 0.01 \\
\hline Dialysis & $527(3)$ & $151(3)$ & 0.78 \\
\hline Chronic kidney disease & 8009 (45) & $2201(42)$ & $<0.001$ \\
\hline Chronic obstructive pulmonary disease & $8283(46)$ & $2436(46)$ & 0.93 \\
\hline Cancer (except non-melanoma skin cancer) & $3020(17)$ & $898(17)$ & 0.79 \\
\hline Metastatic cancer & $566(3)$ & $100(2)$ & $<0.001$ \\
\hline Diabetes & $8648(48)$ & $2800(53)$ & $<0.001$ \\
\hline Median (IQR) systolic blood pressure (SBP) & $133(116-152)$ & $130(115-146)$ & $<0.001$ \\
\hline SBP missing & $9230(52)$ & $70(1)$ & - \\
\hline Median (IQR) serum sodium & $138(136-141)$ & $138(136-140)$ & 0.004 \\
\hline Sodium missing & $12032(67)$ & $29(1)$ & - \\
\hline $\begin{array}{l}\text { Median (IQR) serum B type natriuretic } \\
\text { peptide (BNP) }\end{array}$ & $1249(657-2258)$ & $760(336-1590)$ & $<0.001$ \\
\hline BNP missing & $12810(72)$ & $3167(60)$ & - \\
\hline Median (IQR) serum creatinine (SCr) & $1.3(1.0-1.8)$ & $1.2(1.0-1.6)$ & $<0.001$ \\
\hline SCrmissing & $11488(64)$ & $22(0)$ & - \\
\hline Median (IQR) estimated glomerular filtration rate & $47(32-64)$ & $56(41-72)$ & $<0.001$ \\
\hline
\end{tabular}

$\mathrm{IQR}=$ interquartile range

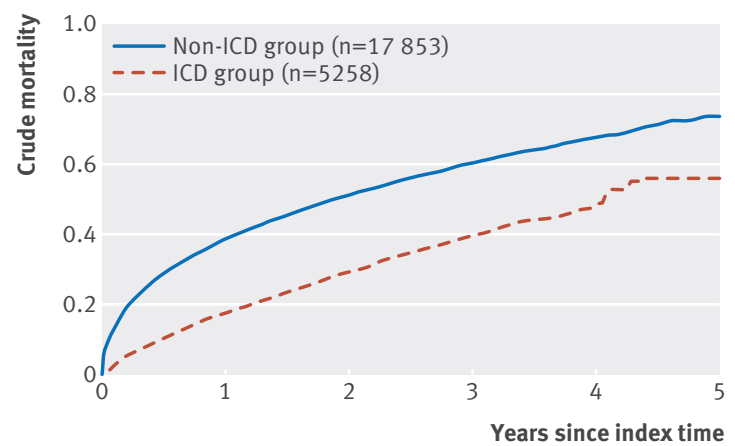

Fig 3 | Crude mortality curves for patients with heart failure with $(n=5258)$ and without implantable cardioverter defibrillator (ICD) $(n=17853)$ in primary cohort

the mortality seen at three years in trials of ICDs in ambulatory recipients. ${ }^{67}$

\section{Effectiveness of ICDs}

After adjustment for bias with latency analyses and the high dimension propensity score, patients who received an ICD during an acute admission for heart failure or other co-morbidity did not have a substantially different risk of mortality (hazard ratio $0.91,95 \%$ confidence interval 0.82 to 1.00$)$ or sudden cardiac death (0.95, 0.78 to 1.17) than those who had no ICD during their admission (table 2, fig 4). This trend remained when we extended the latency period to 365 days (table 2 ) and when the analyses were restricted to the smaller secondary cohort (table 2, fig 4). This trend was also similar in the sensitivity analyses (table 3).

\section{Effectiveness of ICDs in demographic subgroups}

The proportion of eligible patients who received ICDs varied among demographic subgroups with notable differences in age and sex. Women and the oldest patients made up smaller fractions of recipients of ICDs: $14 \%$ women versus $29 \%$ men, and $12 \%$ in the $\geq 81$ age group versus $28-34 \%$ in other age groups (table 4 ). This suggests that ICD implantation might have been more selective among these groups. We found no significant differences in ICD effectiveness among most demographic subgroups, except in the small group of patients aged over 81. Among these older patients, ICD use was associated with a lower mortality risk (hazard ratio $0.78,95 \%$ confidence interval 0.65 to 0.93 ) but not with a significant reduction in risk of sudden cardiac death $(0.74,0.52$ to 1.04 ; fig 5 , table 4$)$. The trend was similar when we extended the latency period to 365 days (table 4).

\section{Effectiveness of ICDs in clinical subgroups Effectiveness by myocardial infarction status}

The findings in the subgroups with and without recent myocardial infarction showed similar effectiveness of ICDs (table 5). Among patients with an old myocardial infarction, ICD therapy was associated with a significantly lower risk of mortality (37\% reduction, hazard ratio $0.63,95 \%$ confidence interval 0.45 to 0.86 ) and a 
Table 2 Number of events and incidence rates for death and sudden cardiac death in patients with and without implantable cardioverter defibrillator (ICD) in latency 180 day* and latency 365 day* analyses

\begin{tabular}{|c|c|c|c|c|c|c|}
\hline \multirow[b]{3}{*}{ Group } & \multicolumn{3}{|l|}{ Death } & \multicolumn{3}{|c|}{$\underline{\text { Sudden cardiac death }}$} \\
\hline & \multirow[b]{2}{*}{ Event/IRt } & \multicolumn{2}{|l|}{$\mathrm{HR}(95 \% \mathrm{Cl})$} & \multirow[b]{2}{*}{ Event/IRT } & \multicolumn{2}{|l|}{$\mathrm{HR}(95 \% \mathrm{Cl})$} \\
\hline & & Crude & Adjusted $¥$ & & Crude & Adjusted \\
\hline \multicolumn{7}{|l|}{ Latency 180 day } \\
\hline \multicolumn{7}{|l|}{ Primary cohort } \\
\hline ICD $(n=5258)$ & 1307/159 & 0.69 (0.65 to 0.74$)$ & 0.91 (0.82 to 1.00$)$ & $330 / 40$ & 0.71 (0.62 to 0.82 ) & 0.95 (0.78 to 1.17$)$ \\
\hline No ICD $(n=17$ 853) & $5299 / 241$ & Reference & Reference & $1326 / 60$ & Reference & Reference \\
\hline \multicolumn{7}{|l|}{ Secondary cohort } \\
\hline ICD $(n=412)$ & $125 / 201$ & 0.90 (0.71 to 1.14$)$ & 1.01 (0.79 to 1.29 ) & $40 / 64$ & 1.26 (0.82 to 1.93$)$ & 1.20 (0.71 to 2.00$)$ \\
\hline No ICD $(n=17853)$ & $5299 / 241$ & Reference & Reference & $1326 / 60$ & Reference & Reference \\
\hline \multicolumn{7}{|l|}{ Latency 365 day } \\
\hline \multicolumn{7}{|l|}{ Primary cohort } \\
\hline ICD $(n=5258)$ & $925 / 155$ & 0.73 (0.68 to 0.79$)$ & 0.94 (0.83 to 1.06$)$ & $234 / 39$ & 0.75 (0.65 to 0.87$)$ & $0.96(0.76$ to 1.21$)$ \\
\hline No ICD $(n=17853)$ & $3524 / 220$ & Reference & Reference & $880 / 55$ & Reference & Reference \\
\hline \multicolumn{7}{|l|}{ Secondary cohort } \\
\hline$I C D(n=412)$ & $90 / 203$ & $0.95(0.72$ to 1.27$)$ & 1.02 (0.72 to 1.43$)$ & $28 / 63$ & 1.13 (0.67 to 1.90$)$ & 0.94 (0.49 to 1.80$)$ \\
\hline No ICD $(n=17853)$ & $3524 / 220$ & Reference & Reference & $880 / 55$ & Reference & Reference \\
\hline
\end{tabular}

Table 3 | Primary and sensitivity analyses of ICD effectiveness. Figures are hazard ratios $(95 \% \mathrm{Cl})$ adjusted for high dimension propensity score (hdPS) ${ }^{\star}$ Complete case analyses not conducted in secondary cohort because of smaller size of cohort.

\begin{tabular}{|c|c|c|c|c|c|c|}
\hline & \multicolumn{3}{|c|}{ Primary cohort } & \multicolumn{3}{|c|}{ Secondary cohort } \\
\hline & ICD/no ICD & Death & Sudden cardiac death & ICD/no ICD & Death & $\begin{array}{l}\text { Sudden cardiac } \\
\text { death }\end{array}$ \\
\hline Primary analyses & $5258 / 17853$ & 0.91 (0.82 to 1.00$)$ & 0.95 (0.78 to 1.17$)$ & $412 / 17853$ & 1.01 (0.79 to 1.29$)$ & 1.20 (0.71 to 2.00$)$ \\
\hline hdPS matched analyses & $2254 / 2254$ & 0.92 (0.77 to 1.09$)$ & 1.03 (0.78 to 1.35$)$ & $291 / 291$ & 0.99 (0.72 to 1.37$)$ & 1.50 (0.94 to 2.38) \\
\hline Complete case analyses & $1801 / 3261$ & 0.87 (0.72 to 1.05$)$ & 1.15 (0.79 to 1.66$)$ & $--^{\star}$ & $-^{\star}$ & $-^{\star}$ \\
\hline
\end{tabular}

non-significant $26 \%$ reduction in sudden cardiac death $(0.74,0.40$ to 1.35$)$.

Effectiveness by presence of left bundle branch block ICD use was associated with a lower risk of mortality (hazard ratio $0.64,95 \%$ confidence interval 0.34 to 1.17 ) and sudden cardiac death (0.51, 0.16 to 1.61$)$ among patients with left bundle branch block (table 5); although the confidence intervals were wide.

\section{Effectiveness by serum B type natriuretic peptide status}

A total of 7134 patients had their admission serum B type natriuretic peptide value documented (2091 (40\%) patients with ICD $v 5043$ (28\%) without). Risks of mortality and sudden cardiac death associated with ICD therapy were numerically lower among patients with low serum B type natriuretic peptide (hazard ratios 0.86

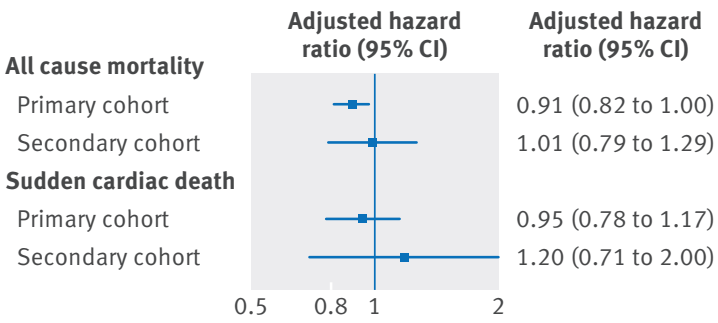

Fig 4 | Hazard ratios (adjusted for high dimension propensity score) for death and sudden cardiac death among primary and secondary cohorts in latency 180 day analyses
(95\% confidence interval 0.67 to 1.10 ) for mortality and 1.11 (0.69 to 1.77) for sudden cardiac death) than those with high type natriuretic peptide (0.94 (0.78 to 1.13 ) and 1.20 (0.85 to 1.69), respectively); however, these risk estimates were not significant (table 5).

\section{Discussion}

\section{Main findings}

The benefits of primary ICD therapy that had been previously shown in ambulatory patients with heart failure do not seem to translate to elderly patients who receive the device during acute hospital admissions for exacerbation of heart failure or other acute co-morbidities. Adjustment for potential confounding and healthy candidate bias ${ }^{11}$ in this specific population reduced the apparent impact of ICD therapy to a $5 \%$ reduction in sudden cardiac death and a 9\% reduction in all cause mortality, which were not significant. This trend remained similar among subgroups of patients aged under 80 and across the sexes and racial subgroups. There was, however, a trend towards benefit of ICDs implanted during acute admissions to hospital in reducing mortality or sudden cardiac death in patients who had non-recent myocardial infarction more than 40 days prior to implantation, left bundle branch block, or lower serum B type natriuretic peptide, although these also did not reach significance.

\section{Strength and limitations}

Our study is the first to use latency analysis to account for the healthy candidate bias in assessing effectiveness 


\begin{tabular}{|c|c|c|c|c|c|c|}
\hline & \multirow{2}{*}{$\begin{array}{l}\text { Sample size } \\
\text { (ICD/no ICD) }\end{array}$} & \multirow{2}{*}{$\begin{array}{l}\% \text { of eligible patients } \\
\text { received ICD }\end{array}$} & \multirow[b]{2}{*}{ Event (ICD/no ICD) } & \multirow[b]{2}{*}{ IRt (ICD/no ICD) } & \multicolumn{2}{|c|}{ Adjusted HR $(95 \% \mathrm{Cl}) \ddagger$} \\
\hline & & & & & Latency 180 day & Latency 365 day \\
\hline \multicolumn{7}{|c|}{ Outcome $=$ death } \\
\hline \multicolumn{7}{|c|}{ Age (years): } \\
\hline $66-70$ & $1322 / 2534$ & 34 & $285 / 568$ & $127 / 137$ & 0.89 (0.70 to 1.13$)$ & $1.02(0.77$ to 1.36$)$ \\
\hline $71-75$ & $1347 / 2950$ & 31 & $301 / 755$ & $136 / 168$ & 1.11 (0.88 to 1.40$)$ & $1.33(1.02$ to 1.74$)$ \\
\hline $76-80$ & $1394 / 3670$ & 28 & $362 / 1068$ & $172 / 216$ & $0.92(0.75$ to 1.12$)$ & $0.82(0.65$ to 1.04$)$ \\
\hline$\geq 81$ & $1195 / 8699$ & 12 & $359 / 2908$ & $213 / 345$ & $0.78 \S(0.65$ to 0.93$)$ & $0.78 \S(0.63$ to 0.96$)$ \\
\hline \multicolumn{7}{|l|}{ Sex: } \\
\hline Men & $3763 / 9321$ & 29 & $945 / 2779$ & $161 / 245$ & 0.92 (0.81 to 1.04) & $0.96(0.82$ to 1.11$)$ \\
\hline Women & $1495 / 8532$ & 14 & $362 / 2520$ & $152 / 237$ & $0.90(0.75$ to 1.07$)$ & $0.90(0.74$ to 1.10$)$ \\
\hline \multicolumn{7}{|l|}{ Race: } \\
\hline White & $4392 / 15068$ & 23 & $1088 / 4402$ & $156 / 241$ & 0.90 (0.81 to 1.01) & $0.96(0.84$ to 1.10$)$ \\
\hline Black & $613 / 1790$ & 26 & $154 / 594$ & $172 / 240$ & $0.93(0.70$ to 1.24$)$ & $0.82(0.57$ to 1.16$)$ \\
\hline Other & $253 / 995$ & 20 & $65 / 303$ & $181 / 240$ & 0.94 (0.60 to 1.47$)$ & 0.84 (0.50 to 1.39$)$ \\
\hline \multicolumn{7}{|c|}{ Outcome $=$ sudden cardiac death } \\
\hline \multicolumn{7}{|c|}{ Age (years): } \\
\hline $66-70$ & $1322 / 2534$ & 34 & $73 / 125$ & $33 / 30$ & 1.04 (0.63 to 1.72$)$ & 1.12 (0.62 to 2.00$)$ \\
\hline $71-75$ & $1347 / 2950$ & 31 & $59 / 176$ & $27 / 39$ & 0.81 (0.48 to 1.35$)$ & 0.73 (0.41 to 1.27$)$ \\
\hline $76-80$ & $1394 / 3670$ & 28 & $99 / 215$ & $47 / 44$ & $1.43(0.95$ to 2.14$)$ & 1.30 (0.82 to 2.07$)$ \\
\hline$\geq 81$ & $1195 / 8699$ & 12 & $99 / 810$ & $59 / 96$ & 0.74 (0.52 to 1.04$)$ & 0.80 (0.54 to 1.18$)$ \\
\hline \multicolumn{7}{|l|}{ Sex: } \\
\hline Men & $3763 / 9321$ & 29 & $248 / 732$ & $42 / 65$ & 1.00 (0.78 to 1.28$)$ & 1.08 (0.82 to 1.42$)$ \\
\hline Women & $1495 / 8532$ & 14 & $82 / 594$ & $35 / 56$ & $0.86(0.59$ to 1.26$)$ & $0.75(0.50$ to 1.15$)$ \\
\hline \multicolumn{7}{|l|}{ Race: } \\
\hline White & $4392 / 15068$ & 23 & $288 / 1121$ & $41 / 61$ & 0.98 (0.79 to 1.23) & 1.06 (0.82 to 1.36$)$ \\
\hline Black & $613 / 1790$ & 26 & $31 / 135$ & $35 / 54$ & 0.77 (0.40 to 1.47$)$ & $0.44(0.20$ to 1.00$)$ \\
\hline Other & $253 / 995$ & 20 & $11 / 70$ & $31 / 55$ & 0.75 (0.24 to 2.33) & 0.60 (0.16 to 2.22) \\
\hline
\end{tabular}

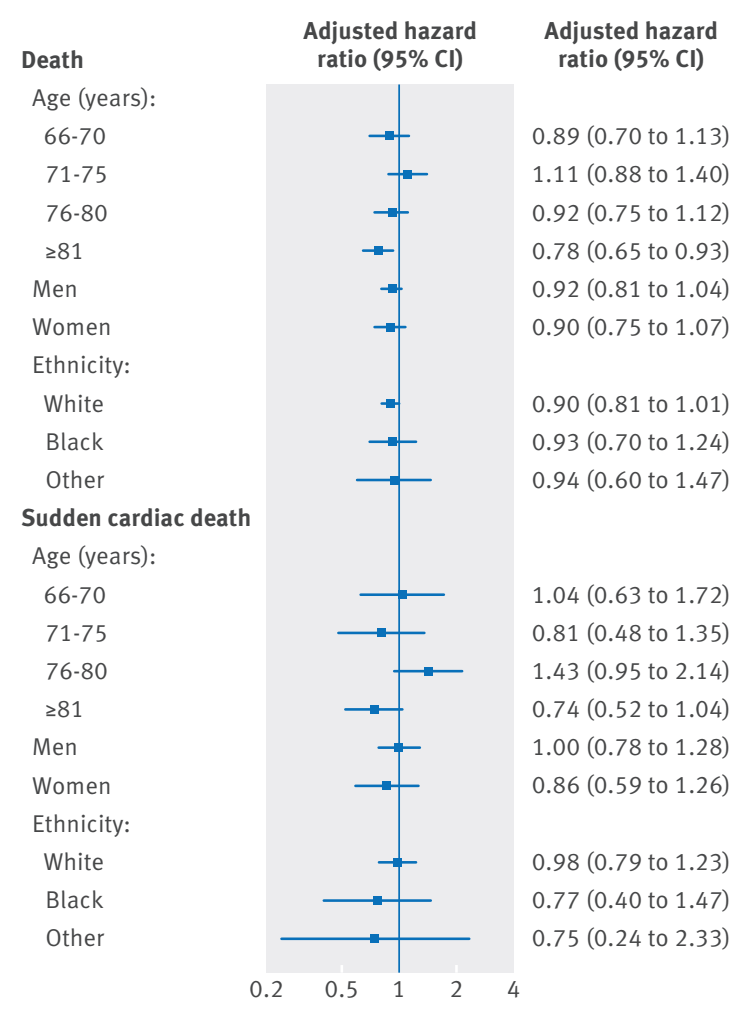

Fig 5 | Hazard ratios (adjusted for high dimension propensity score) for death and sudden cardiac death among demographic subgroups in latency 180 day analyses of ICDs with observational data. Several lines of evidence suggest the existence of healthy candidate bias in the observational studies of ICD. Although the mortality curves of previous randomized trials show no ICD benefit until 1-1.5 years, ${ }^{67}$ we observed an immediate separation of the mortality curves in both our current and previous study, ${ }^{11}$ which is probably caused by healthy candidate bias due to selection of patients for ICD rather than immediate ICD benefit. The existence of healthy candidate bias among patients in hospital has been supported by evidence that ICD recipients had a 40-50\% lower risk of adverse events, such as non-traumatic hip fracture and admission to a skilled nursing facility, than similar patients who did not receive an ICD. ${ }^{11}$ This bias in patient and physician selection for ICD implantation cannot be completely eliminated by adjustment for known risk factors and can lead to overestimation of the net benefit of ICDs. ${ }^{11}$ The disparity between groups is further suggested by a greater difference in total mortality (including non-cardiac death) than in sudden cardiac death, which is the only event that is expected to be reduced by ICDs.

Among strategies that have been developed to account for healthy candidate bias, latency analysis has been shown to be useful25 and is suitable to evaluate ICD effectiveness because of the delayed benefit seen in trials. ${ }^{67}$ This method allows a less biased evaluation by focusing on a time period more likely to reflect true ICD 


\begin{tabular}{|c|c|c|c|c|c|c|}
\hline & \multirow{2}{*}{$\begin{array}{l}\text { Sample size } \\
\text { (ICD/no ICD) }\end{array}$} & \multirow{2}{*}{$\begin{array}{l}\% \text { of eligible patients } \\
\text { received ICD }\end{array}$} & \multirow[b]{2}{*}{ Event (ICD/no ICD) } & \multirow[b]{2}{*}{ IRt (ICD/no ICD) } & \multicolumn{2}{|c|}{ Adjusted HR $(95 \% \mathrm{Cl}) \neq$} \\
\hline & & & & & Latency 180 day & Latency 365 day \\
\hline \multicolumn{7}{|c|}{ Outcome=death } \\
\hline \multicolumn{7}{|c|}{ Myocardial infarction: } \\
\hline Recent & $1685 / 8160$ & 17 & $565 / 4476$ & $165 / 333$ & 0.92 (0.77 to 1.09) & $0.94(0.76$ to 1.16$)$ \\
\hline Old & $448 / 904$ & 33 & $186 / 648$ & $226 / 532$ & $0.63 \S(0.45$ to 0.86$)$ & 0.74 (0.51 to 1.08) \\
\hline No & $3125 / 8789$ & 26 & $1108 / 5317$ & $172 / 369$ & $0.90(0.79$ to 1.03$)$ & 0.91 (0.78 to 1.07) \\
\hline \multicolumn{7}{|c|}{ Left bundle branch block: } \\
\hline No & $5143 / 17406$ & 23 & $1809 / 10161$ & $173 / 358$ & 0.92 (0.83 to 1.01) & 0.94 (0.83 to 1.06) \\
\hline Yes & $115 / 447$ & 20 & $50 / 280$ & $213 / 401$ & 0.64 (0.34 to 1.17) & 0.75 (0.37 to 1.49) \\
\hline \multicolumn{7}{|c|}{ B type natriuretic peptide: } \\
\hline$<800$ & $1085 / 1604$ & 40 & $359 / 905$ & $157 / 302$ & $0.86(0.67$ to 1.10$)$ & $0.86(0.65$ to 1.15$)$ \\
\hline$\geq 800$ & $1006 / 3439$ & 23 & $490 / 2373$ & $278 / 464$ & 0.94 (0.78 to 1.13) & $0.91(0.72$ to 1.13$)$ \\
\hline \multicolumn{7}{|c|}{ Outcome=sudden cardiac death } \\
\hline \multicolumn{7}{|c|}{ Myocardial infarction: } \\
\hline Recent & $1685 / 8160$ & 17 & $115 / 542$ & $34 / 40$ & 1.03 (0.76 to 1.39) & 0.92 (0.64 to 1.33$)$ \\
\hline Old & $448 / 904$ & 33 & $27 / 83$ & $33 / 68$ & $0.74(0.40$ to 1.35$)$ & 1.12 (0.56 to 2.21) \\
\hline No & $3125 / 8789$ & 26 & $188 / 701$ & $29 / 49$ & 0.91 (0.70 to 1.18$)$ & $1.00(0.73$ to 1.38$)$ \\
\hline \multicolumn{7}{|c|}{ Left bundle branch block: } \\
\hline No & $5143 / 17406$ & 23 & $325 / 1289$ & $31 / 45$ & 0.97 (0.80 to 1.17) & $1.02(0.81$ to 1.29$)$ \\
\hline Yes & $115 / 447$ & 20 & $5 / 37$ & $21 / 53$ & $0.51(0.16$ to 1.61$)$ & 0.42 (0.09 to 2.05) \\
\hline \multicolumn{7}{|c|}{ B type natriuretic peptide: } \\
\hline$<800$ & $1085 / 1604$ & 40 & $92 / 199$ & $40 / 66$ & 1.11 (0.69 to 1.77) & $1.42(0.82$ to 2.46$)$ \\
\hline$\geq 800$ & $1006 / 3439$ & 23 & $132 / 539$ & $75 / 105$ & 1.20 (0.85 to 1.69$)$ & 1.17 (0.76 to 1.80) \\
\hline
\end{tabular}

effectiveness rather than preferential selection of ICDs for and by healthier patients. A few caveats should be noted. Healthy candidate bias can continue to influence outcomes beyond the initial chosen latency period, which is likely given our conservative 180 day latency period. Therefore, our latency analysis might still overestimate ICD survival benefit. Additionally, latency analyses could underestimate the survival benefit of ICDs if lifesaving events occurred more frequently during the prespecified latency period. Nevertheless, trial data have indicated such an underestimation is likely minimal. ${ }^{67}$

Several limitations need to be considered when our findings are interpreted. First, and foremost, our findings are not applicable to elderly patients who would undergo ICD implantation electively as outpatients. Our study population was limited to elderly patients who received ICDs during acute admissions to hospital for reasons other than ICD implantation. We selected this population for analysis of effectiveness, as in a previous study by Hernandez and colleagues, ${ }^{39}$ because data are available for comparison of similar patients admitted with heart failure who did not receive ICDs. Many elderly patients, however, receive the device as an elective procedure. The effectiveness of ICD among these healthier patients is more likely to be comparable with that of the trials on which the guideline recommendations are based. Additionally, our findings cannot be generalized to patients who received cardiac resynchronization therapy with their ICD (CRT-D) as this is likely to decrease heart failure events. We also did not include patients who received the ICD as secondary prevention, for which lifesaving ICD therapies are more likely to occur.

We could not identify all patients with a history of myocardial infarction, left bundle branch block, or low serum B type natriuretic peptide values because we used a claims based definition and because of missing data on B type natriuretic peptide. Therefore, we did not have a sufficient sample size to confirm the trends seen for those subgroups thought to derive more benefit from ICDs. ${ }^{30-3335}$ Neither did we have complete information on all the recognized risk factors for death from heart failure, such as the New York Heart Association class and duration of QRS. Residual confounding is possible. Regarding the general problem of missing values in registry variables, our results were robust to the missingness assumption, as the results based on imputed datasets were similar to complete case analyses. ${ }^{40}$

\section{Comparison with other studies}

Our study of patients admitted to hospital failed to show survival benefits of primary ICD therapy similar to those seen in trials of healthier ambulatory patients, in whom there was a $23-31 \%$ reduction in mortality ${ }^{67}$; this is likely explained by differences in the patient population. The median age of the SCDHeFT population was 60 and the mean age of the MADIT II population was 64.67 The patients in our study were older, with a mean age of 75 in the ICD recipients and 80 in those who did not receive an ICD. It is not clear that age alone is the major difference as benefit has been shown in subsets of elderly patients 
in randomized trials. ${ }^{41}$ More importantly, previous trials were conducted among ambulatory patients with symptoms of stable mild-to-moderate heart failure, many of whom had not previously been admitted to hospital with heart failure. Our study focused on a population of patients admitted for exacerbation of heart failure or other acute causes. This particular subset of patients has a higher baseline burden of heart failure and other co-morbidities than trial populations. Older age, ${ }^{42-44}$ advanced heart failure, ${ }^{424546}$ and non-cardiac co-morbidities ${ }^{46}$ increase the likelihood of mortality that will not be prevented by an ICD, and thus present competing risks for prolonged survival with an ICD.

Four previous studies reported that primary ICD implantation in routine clinical practice was associated with a sizable survival benefit comparable with those seen in major trials, ${ }^{3947-49}$ but only one ${ }^{39}$ included the subset of elderly patients who underwent device implantation during an acute hospital admission. In addition, two of these studies enrolled participants from outpatient cardiology clinics, where the ICD was generally implanted as an elective procedure. 4849 The previous observational studies 394849 also found immediate separation of survival curves at a point in time unlikely to be strongly influenced by ICDs.

\section{Implications for practice and future research}

Our results extend the understanding of the clinical effectiveness of ICDs in elderly patients admitted to hospital, who are not typical of patients in trials. Patients admitted for acute exacerbation of heart failure or other co-morbidities might be at greater risk both during ICD implantation and for non-arrhythmic events after discharge. For early risk, it is possible that there is some similarity between patients admitted for heart failure and patients early after myocardial infarction, who also showed no benefit from ICD and an increased risk of non-sudden cardiac death. ${ }^{30} 3150$ For patients non-electively admitted to hospital for heart failure or their comorbidities, it might be appropriate to delay the decision to implant a primary ICD until they have been discharged and can be re-evaluated in the outpatient setting.

Our findings provide no reason to restrict access to ICDs for older patients with heart failure who otherwise seem similar to patients in pivotal ICD trials. The subset of elderly patients who received primary prevention ICDs in trials in outpatient settings were previously shown to derive benefit. ${ }^{41}$ While an ideal study would be a randomized trial in elderly patients, this is not likely to be performed in time to inform imminent clinical decision making. We failed to show a benefit only for those older patients receiving ICDs during an urgent admission for exacerbation of heart failure or other acute causes. Future research is warranted to identify other groups of older patients who have a high or low likelihood of benefit from ICDs to maximize the lifesaving potential of their use.
The efficacy of ICDs in women has also been questioned, in large part because they were under-represented in previous trials. ${ }^{6751}$ In our study of 10027 eligible women, we observed no heterogeneity in effectiveness of ICDs between the sexes; however, it has been shown that female candidates for primary ICD are likely have a lower risk of sudden cardiac death than male candidates. ${ }^{52}$ Women also more often experience complications with ICDs. ${ }^{534}$ Thus, the benefit-risk equation of ICDs among women might require further investigation.

The higher survival associated with ICDs in patients aged over 81 further emphasizes the likelihood of residual bias that cannot be adjusted for using currently reported patient characteristics. The oldest group of patients in our study comprised the lowest proportion of ICD recipients out of eligible recipients. This probably indicates a particularly rigorous selection of healthy ICD recipients in this age group, excluding patients with obvious co-morbidities and more general frailty. Therefore, despite the use of latency analysis and adjustment for high dimension propensity scores, accounting for patient and physician selection is still challenging in assessing real world clinical effectiveness.

Shared decision making regarding primary prevention ICD has been recommended to involve explicit consideration of patient preferences and the likelihood of competing risks for mortality in all patients. ${ }^{55}$ These decisions require particular scrutiny for patients admitted to hospital for exacerbation of heart failure or other acute causes. Recognition of those patients whose dominant risks are from decompensated heart failure and non-cardiac co-morbidities will allow for focused ICD therapy in those patients for whom the device offers the most benefit to provide meaningful prolonging of life.

We thank Jeptha Curtis of Yale School of Medicine and Yale-New Haven Hospital, Sherri Dodd of Medtronic, Kenneth Ellenbogen of Virginia Commonwealth University School of Medicine, Marcel E Salive of the National Institute of Aging at National Institutes of Health, and Lynette Voshage-Stahl of Boston Scientific for the guidance and expertise they contributed to this project by serving on the technical expert panel. We also thank Drew Pratt of the National Cancer Institute at the National Institutes of Health for his review of the manuscript. The views expressed in this article are those of the authors and do not represent opinions of the panel members.

This article was presented in part at the AHA Scientific Sessions 2013 on 18 November 2013 in Dallas, TX, and at the 2014 International Conference of Pharmacoepidemiology on 25 October 2014 in Taipei, Taiwan.

The views expressed in this article are those of the authors and do not represent policies of the AHRQ, CMS or the US DHHS. This manuscript was prepared while CYC was employed at Brigham and Women's Hospital/Harvard Medical School. CYC is now a visiting scientist at the Division of Epidemiology II, Office of Pharmacovigilance and Epidemiology, Office of Surveillance and Epidemiology, Center for Drug Evaluation and Research, US Food and Drug Administration. The opinions expressed in this work are the author's own and do not reflect the view of the Food and Drug Administration, the Department of Health and Human Services, or the US government.

Contributors: SS and IWS contributed to the acquisition of the data. C-YC, SS and LWS developed the study design. C-YC, SS, and MD contributed to the analysis of the data. C-YC and SS drafted the manuscript. All authors contributed to the interpretation of the data 
and the revision of the work, and all approved the final version to be published. CYC and SS are the guarantors. The authors of this report are responsible for its content. Statements in the report should not be construed as endorsement by the Agency for Healthcare Research and Quality or the US Department of Health and Human Services.

Funding: This project is funded by contract No HHSA290-20050016-I -TO3 from the Agency for Healthcare Research and Quality (AHRQ), US Department of Health and Human Services (DHHS) as part of the Developing Evidence to Inform Decisions about Effectiveness (DEcIDE) program, IAA Contract 500-2010-00001I TO6 and CEA Contract 500-2010-00001I TO2 from the Centers for Medicare and Medicaid Services (CMS), US DHHS. The funding agency had no role in the design and conduct of the study and in the collection, analysis, and interpretation of the data. The manuscript was based on a report done under contract to AHRQ; AHRQ had the draft report reviewed by independent peer reviewers before acceptance of the final report.

Competing interests: All authors have completed the ICMJE uniform disclosure form at http://www.icmje.org/coi_disclosure.pdf (available on request from the corresponding author) and declare no financial relationships with any organizations that might have an interest in the submitted work in the previous 3 years; no other relationships or activities that could appear to have influenced the submitted work. SS is supported by a mid-career development award grant K02-HS017731 from the AHRQ, US DHHS. She also reported receiving research support from Johnson and Johnson and receiving personal income for consulting from Sanofi-Aventis. SS has made available online a detailed listing of financial disclosures (http:// www.dcri.duke.edu/about-us/conflict-of-interest/). JDS is a paid consultant to Optum Insight and WHISCON. DBL discloses the following relationship - advisory board: Cardax, Elsevier Practice Update Cardiology, Medscape Cardiology, Regado Biosciences; board of directors: Boston VA Research Institute, Society of Cardiovascular Patient Care; chair: American Heart Association Get With The Guidelines Steering Committee; data monitoring committees: Duke Clinical Research Institute, Harvard Clinical Research Institute, Mayo Clinic, Population Health Research Institute; honorariums: American College of Cardiology (senior associate editor, Clinical Trials and News, ACC.org), Belvoir Publications (editor in chief, Harvard Heart Letter), Duke Clinical Research Institute (clinical trial steering committees), Harvard Clinical Research Institute (clinical trial steering committee), HMP Communications (editor in chief, Journal of Invasive Cardiology), Journal of the American College of Cardiology (associate editor), Population Health Research Institute (clinical trial steering committee), Slack Publications (Chief Medical Editor, Cardiology Today's Intervention), WebMD (CME steering committees); other: Clinical Cardiology (deputy editor); research funding: Amarin, AstraZeneca, Biotronik, Bristol-Myers Squibb, Eisai, Ethicon, Forest Laboratories, Ischemix, Medtronic, Pfizer, Roche, Sanofi Aventis, St. Jude Medical, The Medicines Company; trustee: American College of Cardiology; unfunded research: FlowCo, PLx Pharma, Takeda.

Ethical approval: This study was approved by the institutional review board of Brigham and Women's Hospital (IRB No 2009P002819).

Transparency: The lead authors, CYC and SS, affirm that this manuscript is an honest, accurate, and transparent account of the study being reported; that no important aspects of the study have been omitted; and that any discrepancies from the study as planned (and, if relevant, registered) have been explained.

Data sharing: No additional data available.

This is an Open Access article distributed in accordance with the Creative Commons Attribution Non Commercial (CC BY-NC 4.0) license, which permits others to distribute, remix, adapt, build upon this work non-commercially, and license their derivative works on different terms, provided the original work is properly cited and the use is noncommercial. See: http://creativecommons.org/licenses/by-nc/4.0/.

1 Mond HG, Proclemer A. The 11th world survey of cardiac pacing and implantable cardioverter-defibrillators: calendar year 2009-a World Society of Arrhythmia's project. Pacing Clin Electrophysiol 2011:34:1013-27.

2 Hammill SC, Kremers MS, Stevenson LW, et al. Review of the registry's fourth year, incorporating lead data and pediatric ICD procedures, and use as a national performance measure. Heart Rhythm 2010;7:1340-5

3 Proclemer A, Ghidina M, Gregori D, P et al. Impact of the main implantable cardioverter-defibrillator trials in clinical practice: data from the Italian ICD Registry for the years 2005-07. Europace 2009; $11: 465-75$
4 Heidenreich PA, Albert NM, Allen LA, et al. Forecasting the impact of heart failure in the United States: a policy statement from the American Heart Association. Circ Heart Fail 2013;6:606-19.

5 Stewart S, MacIntyre K, Capewell S, McMurray JJ. Heart failure and the aging population: an increasing burden in the 21st century? Heart 2003;89:49-53.

6 Bardy GH, Lee KL, Mark DB, et al. Amiodarone or an implantable cardioverter-defibrillator for congestive heart failure. N Engl I Med 2005;352:225-37.

7 Moss Al, Zareba W, Hall W], et al. Prophylactic implantation of a defibrillator in patients with myocardial infarction and reduced ejection fraction. N Engl J Med 2002;346:877-83.

8 Chen CY, Stevenson LW, Stewart GC, et al. Impact of baseline heart failure burden on post-implantable cardioverter-defibrillator mortality among medicare beneficiaries. J Am Coll Cardiol 2013;61:2142-50.

9 Stewart G, Chen CY, Stevenson LW, et al. Outcomes among Medicare beneficiaries are optimized when primary ICD implant occurs during an elective rather than unplanned hospitalization. Circulation 2013;128:A11117.

10 Solomon SD, Dobson J. Pocock S, et al. Influence of nonfatal hospitalization for heart failure on subsequent mortality in patients with chronic heart failure. Circulation 2007;116:1482-7.

11 Setoguchi S, Warner SL, Stewart GC, et al. Influence of healthy candidate bias in assessing clinical effectiveness for implantable cardioverter-defibrillators: cohort study of older patients with heart failure. BMJ 2014;348:g2866.

12 Hammill SC, Stevenson LW, Kadish AH, et al. Review of the registry's first year, data collected, and future plans. Heart Rhythm 2007;4:1260-3.

13 Hammill SC, Kremers MS, Stevenson LW, et al. Review of the registry's second year, data collected, and plans to add lead and pediatric ICD procedures. Heart Rhythm 2008;5:1359-63.

14 Hammill SC, Kremers MS, Kadish AH, et al. Review of the ICD registry's third year, expansion to include lead data and pediatric ICD procedures, and role for measuring performance. Heart Rhythm 2009;6:1397-401.

15 Messenger JC, Ho KK, Young CH, et al. The National Cardiovascular Data Registry (NCDR) data quality brief: the NCDR data quality program in 2012. J Am Coll Cardiol 2012;60:1484-8.

16 Albert NM, Yancy CW, Liang L, et al. Use of aldosterone antagonists in heart failure. JAMA 2009:302:1658-65.

17 Patel UD, Hernandez AF, Liang L, et al. Quality of care and outcomes among patients with heart failure and chronic kidney disease: a Get With the Guidelines-Heart Failure Program study. Am Heart J 2008;156:674-81.

18 Setoguchi S, Zhu Y, Jalbert JJ, Williams LA, Chen CY. Validity of deterministic record linkage using multiple indirect personal identifiers: linking a large registry to claims data. Circ Cardiovasc Qual Outcomes 2014;7:475-80

19 Epstein AE, DiMarco JP, Ellenbogen KA, et al. ACC/AHA/HRS 2008 guidelines for device-based therapy of cardiac rhythm abnormalities: a report of the American College of Cardiology/ American Heart Association Task Force on Practice Guidelines (Writing Committee to Revise the ACC/AHA/NASPE 2002 Guideline Update for Implantation of Cardiac Pacemakers and Antiarrhythmia Devices): developed in collaboration with the American Association for Thoracic Surgery and Society of Thoracic Surgeons. Circulation 2008;117:e350-e408.

20 Strickberger SA, Conti J, Daoud EG, et al. Patient selection for cardiac resynchronization therapy: from the Council on Clinical Cardiology Subcommittee on Electrocardiography and Arrhythmias and the Quality of Care and Outcomes Research Interdisciplinary Working Group, in collaboration with the Heart Rhythm Society. Circulation 2005;111:2146-50

21 Tracy CM, Epstein AE, Darbar D, et al. 2012 ACCF/AHA/HRS focused update of the 2008 guidelines for device-based therapy of cardiac rhythm abnormalities: a report of the American College of Cardiology Foundation/American Heart Association Task Force on Practice Guidelines and the Heart Rhythm Society. [corrected]. Circulation 2012;126:1784-800

22 Chung CP, Murray KT, Stein CM, Hall K, Ray WA. A computer case definition for sudden cardiac death. Pharmacoepidemiol Drug Saf 2010;19:563-72.

23 Centers for Disease Control and Prevention. National Death Index. CDC, 2014. www.cdc.gov/nchs/ndi.htm.

24 Arrighi HM, Hertz-Picciotto I. The evolving concept of the healthy worker survivor effect. Epidemiology 1994;5:189-96.

25 Arrighi HM, Hertz-Picciotto I. Controlling the healthy worker survivor effect: an example of arsenic exposure and respiratory cancer. Occup Environ Med 1996;53:455-62

26 Thygesen LC, Hvidtfeldt UA, Mikkelsen S, Bronnum-Hansen H. Quantification of the healthy worker effect: a nationwide cohort study among electricians in Denmark. BMC Public Health 2011;11:571.

27 Schneeweiss S, Rassen JA, Glynn RJ, et al. High-dimensional propensity score adjustment in studies of treatment effects using health care claims data. Epidemiology 2009;20:512-22. 
28 Rassen JA, Doherty M, Huang W, Schneeweiss S. Pharmacoepidemiology toolbox. BWH, 2014. www.drugepi.org/ dope-downloads/\#PharmacoepidemiologyToolbox.

29 Little R, Rubin D. Statistical analysis with missing data. John Wiley, 2002.

30 Hohnloser SH, Kuck KH, Dorian P, et al. Prophylactic use of an implantable cardioverter-defibrillator after acute myocardial infarction. N Engl J Med 2004;351:2481-8.

31 Steinbeck G, Andresen D, Seidl K, et al. Defibrillator implantation early after myocardial infarction. N Engl J Med 2009;361:1427-36.

32 Abdel-Qadir HM, Tu JV, Austin PC, Wang JT, Lee DS. Bundle branch block patterns and long-term outcomes in heart failure. Int J Cardiol 2011;146:213-8

33 Baldasseroni S, Opasich C, Gorini M, et al. Left bundle-branch block is associated with increased 1-year sudden and total mortality rate in 5517 outpatients with congestive heart failure: a report from the Italian network on congestive heart failure. Am Heart I 2002;143:398-405.

34 Kiyota Y, Schneeweiss S, Glynn RJ, Cannuscio CC, Avorn J, Solomon DH. Accuracy of Medicare claims-based diagnosis of acute myocardial infarction: estimating positive predictive value on the basis of review of hospital records. Am Heart J 2004;148:99-104.

35 Santaguida PL, Don-Wauchope AC, Oremus M, et al. BNP and NT-proBNP as prognostic markers in persons with acute decompensated heart failure: a systematic review. Heart Fail Rev 2014;19:453-70

36 Nunez J, Nunez E, Robles R, et al. Prognostic value of brain natriuretic peptide in acute heart failure: mortality and hospital readmission. Rev Esp Cardiol 2008;61:1332-7.

37 Fonarow GC, Peacock WF, Phillips CO, Givertz MM, Lopatin M. Admission B-type natriuretic peptide levels and in-hospital mortality in acute decompensated heart failure. J Am Coll Cardio 2007;49:1943-50

38 Rassen JA, Glynn RJ, Rothman KJ, Setoguchi S, Schneeweiss S. Applying propensity scores estimated in a full cohort to adjust for confounding in subgroup analyses. Pharmacoepidemiol Drug Saf 2012;21:697-709.

39 Hernandez AF, Fonarow GC. Hammill BG, et al. Clinical effectiveness of implantable cardioverter-defibrillators among medicare beneficiaries with heart failure. Circ Heart Fail 2010;3:7-13.

40 Sterne JA, White IR, Carlin JB, et al. Multiple imputation for missing data in epidemiological and clinical research: potential and pitfalls. BMJ 2009;338:b2393.

41 Huang DT, Sesselberg HW, McNitt S, WJ et al. Improved survival associated with prophylactic implantable defibrillators in elderly patients with prior myocardial infarction and depressed ventricula function: a MADIT-II substudy. J Cardiovasc Electrophysiol 2007; $18: 833-8$

42 Effect of metoprolol CR/XL in chronic heart failure: Metoprolol CR/XL Randomised Intervention Trial in Congestive Heart Failure (MERIT-HF). Lancet 1999;353:2001-7.

43 Cleland JG, Thygesen K, Uretsky BF, et al. Cardiovascular critical even pathways for the progression of heart failure; a report from the ATLAS study. Eur Heart / 2001;22:1601-12.

44 Mehta PA, Dubrey SW, McIntyre HF, et al. Mode of death in patients with newly diagnosed heart failure in the general population. Eur J Heart Fail 2008;10:1108-16.

45 Mozaffarian D, Anker SD, Anand I, et al. Prediction of mode of death in heart failure: the Seattle Heart Failure Model. Circulation 2007;116:392-8.
46 Setoguchi S, Nohria A, Rassen JA, Stevenson LW, Schneeweiss S. Maximum potential benefit of implantable defibrillators in preventing sudden death after hospital admission because of heart failure. CMA 2009;180:611-6.

47 Al-Khatib SM, Hellkamp AS, Fonarow GC, et al. Association between prophylactic implantable cardioverter-defibrillators and survival in patients with left ventricular ejection fraction between 30\% and 35\%. JAMA 2014;311:2209-15.

48 Chan PS, Chow T, Kereiakes D, et al. Effectiveness of implantable cardioverter-defibrillators in patients with ischemic heart disease and left ventricular dysfunction. Arch Intern Med 2006;166: 2228-33.

49 Chan PS, Nallamothu BK, Spertus JA, et al. Impact of age and medical co-morbidity on the effectiveness of implantable cardioverterdefibrillators for primary prevention. Circ Cardiovasc Qual Outcomes 2009;2:16-24.

50 Dorian P, Hohnloser SH, Thorpe KE, et al. Mechanisms underlying the lack of effect of implantable cardioverter-defibrillator therapy on mortality in high-risk patients with recent myocardial infarction: insights from the Defibrillation in Acute Myocardial Infarction Trial (DINAMIT). Circulation 2010;122:2645-52

51 Bristow MR, Saxon LA, Boehmer J, et al. Cardiac-resynchronization therapy with or without an implantable defibrillator in advanced chronic heart failure. N Engl / Med 2004;350):2140-50.

52 Rho RW, Patton KK, Poole JE, et al. Important differences in mode of death between men and women with heart failure who would qualify for a primary prevention implantable cardioverter-defibrillator. Circulation 2012;126:2402-7.

53 MacFadden DR, Crystal E, Krahn AD, et al. Sex differences in implantable cardioverter-defibrillator outcomes: findings from a prospective defibrillator database. Ann Intern Med 2012;156:195-203.

54 Peterson PN, Daugherty SL, Wang Y, et al. Gender differences in procedure-related adverse events in patients receiving implantable cardioverter-defibrillator therapy. Circulation 2009;119:1078-84

55 Yancy CW, Jessup M, Bozkurt B, et al. 2013 ACCF/AHA guideline for the management of heart failure: executive summary: a report of the American College of Cardiology Foundation/American Heart Association Task Force on practice guidelines. Circulation 2013;128:1810-52.

(C) BMJ Publishing Group Ltd 2015

Appendix 1: Detailed description of the databases and data linkage

Appendix 2: Codes for underlying cause of death diagnosis for considering as sudden cardiac death cases

Appendix 3: Description of National Death Appendix 4: Potential predictors of missing values in the imputation model

Appendix 5: Main baseline characteristics by exposure status in secondary cohort 